\title{
DETERMINAÇÃO DE 3,4-METILENODIOXIMETANFETAMINA (MDMA) EM COMPRIMIDOS DE ECSTASY POR CROMATOGRAFIA LÍQUIDA DE ALTA EFICIÊNCIA COM DETECÇÃO POR FLUORESCÊNCIA (CLAE-DF)
}

\author{
José Luiz da Costa*, Estela Regina Pintao, Célia Maria Castro Corrigliano e Osvaldo Negrini Neto \\ Centro de Exames, Análises e Pesquisas, Instituto de Criminalística, Superintendência da Polícia Técnico Científica do Estado de \\ São Paulo, R. Moncorvo Filho, 410, 05507-060 São Paulo - SP, Brasil
}

Recebido em 17/6/08; aceito em 28/10/08; publicado na web em 26/2/09

\begin{abstract}
DETERMINATION OF 3,4-METHYLENEDIOXYMETHAMPHETAMINE (MDMA) IN ECSTASY TABLETS BY HIGH PERFORMANCE LIQUID CHROMATOGRAPHY WITH FLUORESCENCE DETECTION (HPLC-FD). This paper describes the development and validation of simple and selective analytical method for determination of 3.4-methylenedioxymethamphetamine (MDMA) in Ecstasy tablets, using high performance liquid chromatography with fluorescence detection. Analysis was performed in a reversed phase column (LiChrospher $100 \mathrm{C} 18,150 \times 4.6 \mathrm{~mm}, 5 \mu \mathrm{m}$ ), isocratic elution with phosphate buffer $25 \mathrm{mmol} / \mathrm{L} \mathrm{pH} 3.0$ and acetonitrile $(95: 5, \mathrm{v} / \mathrm{v})$. The method presents adequate linearity, selectivity, precision and accuracy. MDMA concentration in analyzed tablets showed a remarkable variability (from 8.5 to $59.5 \mathrm{mg} /$ tablet) although the tablet weights were uniform, indicating poor manufacturing control thus imposing additional health risks to the users.
\end{abstract}

Keywords: HPLC; MDMA; drugs of abuse.

\section{INTRODUÇÃO}

As drogas sintéticas estão entre as substâncias controladas mais consumidas no ocidente, sendo as principais representantes desta classe a 3,4-metilenodioximetanfetamina (MDMA, Ecstasy), a 3,4-metilenodioxietilanfetamina (MDEA, Eve) e a 3,4-metilenodioxianfetamina (MDA) (Figura 1). Estas substâncias são estruturalmente semelhantes à anfetamina e a algumas fenilaquilaminas de atividade alucinógena. Contudo, a atividade farmacológica destas substâncias não se restringe apenas à simples atividade estimulante ou alucinógena, sendo classificada por alguns autores como entactógenos, nome que designaria substâncias cujos efeitos estariam relacionados com a indução de um estado emocional agradável, com aumento da empatia, comunicabilidade e sociabilidade. ${ }^{1}$

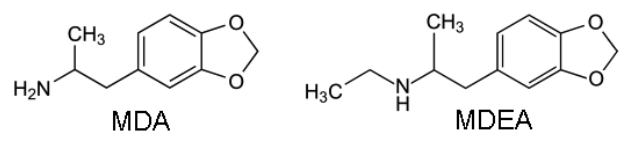<smiles>CNC(C)Cc1ccc2c(c1)OCO2</smiles>

Figura 1. Estrutura química da MDMA e seus análogos estruturais

O consumo e o tráfico internacional de Ecstasy aumentou geometricamente desde a década de 90. Dados da Organização das Nações Unidas (ONU) reportam que existiam em 2006 aproximadamente 24,7 milhões de usuários de estimulantes do grupo anfetamínico (amphetamine-type stimulants), equivalente a $0,6 \%$ da população mundial com idade entre 15 e 64 anos. Deste grupo, 9 milhões seriam usuários de Ecstasy. ${ }^{2}$ Esses dados refletiram que o consumo mundial de drogas sintéticas já superou o de drogas como cocaína e heroína. ${ }^{2}$

*e-mail: jose.jlc@polcientifica.sp.gov.br
A MDMA já ocupa o segundo lugar no ranking de consumo de drogas na maioria dos países europeus, sendo superada somente pela maconha. ${ }^{3}$ Durante o ano 2000 a Europol (European Police Office) apreendeu 17,4 milhões de comprimidos de Ecstasy nos países membros da União Européia, o que corresponde a um aumento de $50 \%$ quando comparado com o número de apreensões que aconteceram durante o ano de $1999 .{ }^{4}$ No Brasil, é crescente sua divulgação pela mídia e o uso recreacional tem sido identificado em vários pacientes que buscam tratamento para farmacodependência nas clínicas de São Paulo. ${ }^{5-7}$ Informações fornecidas pelo Núcleo de Exames em Entorpecentes do Instituto de Criminalística de São Paulo, durante o ano 2001 foram apreendidos 1061 comprimidos de Ecstasy pela Polícia Civil do Estado de São Paulo; no ano seguinte este número foi de 5.677. Durante o período de janeiro a março de 2005, a Polícia Federal apreendeu 31.623 comprimidos da droga no Brasil. ${ }^{8}$

Segundo a ONU, o aumento no número de apreensões de determinada classe de drogas de abuso é indicativo do aumento no consumo desta substância. Mesmo com o aumento no número de apreensões e a notada presença das metilenodioxianfetaminas como fármacos de abuso no Brasil, até o momento não existem informações precisas sobre o consumo destas substâncias. ${ }^{2}$

Este crescente aumento no consumo pode ainda estar associado ao fato de que o tráfico de Ecstasy oferece vantagens quando comparado com drogas tradicionais, como maconha, cocaína e heroína, relacionadas principalmente ao fato de se tratar de uma droga sintética, que não precisa de grande espaço para o cultivo de plantas utilizadas como matéria-prima. Outra vantagem está no fato de que as vias de síntese da MDMA são relativamente simples, amplamente difundidas na internet e não requerem conhecimentos avançados em síntese orgânica.

O Ecstasy é comercializado comumente na forma de comprimidos de bom aspecto, de grande variedade de cores, formas e tamanhos, estampados com vários tipos de figuras e logotipos. À semelhança de outras drogas vendidas no mercado ilícito, não existe controle sobre a composição destes comprimidos, podendo existir grande variação no que diz respeito à quantidade de princípio ativo (MDMA) e à presença 
de adulterantes, substâncias adicionadas ao comprimido para mimetizar e/ou potencializar os efeitos induzidos pelo MDMA., ${ }^{9,10}$

Para análise de MDMA, a cromatografia líquida de alta eficiência é uma técnica analítica freqüentemente citada na literatura internacional. ${ }^{11-15} \mathrm{O}$ detector de fluorescência é, sem dúvida, o mais utilizado na detecção de MDMA e seus análogos após separação no HPLC, pois aproveita-se da fluorescência natural destas substâncias fornecendo análises de alta sensibilidade e especificidade, mesmo em estudos em materiais biológicos complexos como sangue total. ${ }^{13,14}$

O presente trabalho teve por objetivo desenvolver e validar um método analítico para determinar a concentração de MDMA em comprimidos de Ecstasy por cromatografia líquida de alta eficiência com detecção por fluorescência. O método desenvolvido foi aplicado a cinco diferentes grupos de comprimidos de Ecstasy.

\section{PARTE EXPERIMENTAL}

\section{Materiais}

Soluções padrão de MDMA, MDEA e MDA, na concentração de $1 \mathrm{mg} / \mathrm{mL}$ em metanol foram obtidas da Cerilliant (Round Rock, EUA). A água utilizada no preparado da fase móvel foi ultrapurificada em sistema Milli-Q (Millipore, Bedford, EUA). Todos os demais reagentes utilizados eram de pureza adequada para cromatografia líquida.

\section{Amostras}

Foram selecionados para análise 47 comprimidos que foram encaminhados para exame pericial no Núcleo de Análise Instrumental do Instituto de Criminalística - Superintendência da Polícia Técnico Científica do Estado de São Paulo, e que apresentaram resultado positivo para MDMA em ensaios de triagem (reações colorimétricas e/ou cromatografia em camada delgada). Estes comprimidos foram divididos em cinco diferentes grupos, de acordo com suas características físicas (cor, tamanho e formato): grupo 1 - 12 comprimidos de cor laranja, de forma arredondada, de $7 \mathrm{~mm}$ de diâmetro, com uma das faces lisas e a outra apresentando uma figura semelhante a um morcego; grupo 2 - 5 comprimidos de cor branca, de forma arredondada, de $7 \mathrm{~mm}$ de diâmetro, com uma das faces contendo uma fissura mediana e a outra apresentando as inscrições "D\&G"; grupo 3 - 8 comprimidos de cor bege, de forma arredondada, de 9 $\mathrm{mm}$ de diâmetro, com uma das faces lisas e a outra apresentando as inscrições "LV"; grupo 4 - 10 comprimidos de cor branca, de forma arredondada, de $8 \mathrm{~mm}$ de diâmetro, com uma das faces lisas e a outra apresentando as inscrições "TOP" e, grupo 5 - 12 comprimidos de cor branca, de forma arredondada, de $9 \mathrm{~mm}$ de diâmetro, com uma das faces contendo uma fissura mediana e a outra apresentando uma figura semelhante a um pombo.

\section{Equipamentos e condições analíticas}

Foi utilizado equipamento de cromatografia líquida de alta eficiência LaChrom (Merck, Darmstadt, Alemanha) composto por bomba quaternária com sistema de degaseificação da fase móvel (modelo L-7100), injetor automático (modelo L-7200), forno de coluna (modelo L-7300) e detector por fluorescência (modelo L-7485). O controle do equipamento e aquisição dos dados foi feito através do software HSM ${ }^{\circledR}$ (Merck, Darmstadt, Alemanha). As análises foram realizadas utilizando-se coluna cromatográfica LiChrospher ${ }^{\circledR} 100(\mathrm{C}-18,125$ x $4 \mathrm{~mm}$, com partículas de $5 \mu \mathrm{m}$, Merck, Darmstadt, Alemanha), mantida a $30{ }^{\circ} \mathrm{C}$. A fase móvel utilizada foi constituída por mistura de tampão fosfato $25 \mathrm{mM} \mathrm{pH}$ 3,0 e acetonitrila (95:5 v/v), com eluição em modo isocrático, com vazão de 1,0 $\mathrm{mL} / \mathrm{min}$. A detecção do analito valeu-se da capacidade intrínseca desta substância de emitir fluorescência. Por esta razão foi utilizado detector de fluorescência, com comprimento de onda de excitação $\left(\lambda_{\text {ex }}\right)$ e de emissão $\left(\lambda_{\text {em }}\right)$ ajustados em 288 e 324 nm, respectivamente.

\section{Preparo da amostra}

Inicialmente, os comprimidos foram pesados em balança analítica para determinação do peso médio de cada grupo. Após a pesagem, os comprimidos foram pulverizados utilizando-se almofariz de porcelana. A partir do pó obtido, uma alíquota de $20 \mathrm{mg}$ foi pesada e transferida para balão volumétrico com capacidade de $10 \mathrm{~mL}$, ao qual foram adicionados $9 \mathrm{~mL}$ de metanol. O balão foi então submetido à agitação mecânica por 10 min, seguido por banho ultrassônico por mesmo período de tempo, sendo posteriormente completado seu volume com o mesmo solvente. Desta solução, uma alíquota de $1 \mathrm{~mL}$ foi então transferida para o balão volumétrico com capacidade para $100 \mathrm{~mL}$ e o volume completado com água ultrapura. Esta solução foi filtrada através de membrana com 0,45 $\mu \mathrm{m}$ de porosidade e submetida à análise cromatográfica.

\section{Validação}

O método desenvolvido foi validado de acordo com procedimentos e parâmetros de confiança analítica comumente utilizados em toxicologia forense. ${ }^{16,17}$ Durante a validação foram considerados os seguintes parâmetros analíticos: seletividade, linearidade, precisão (repetibilidade e precisão intermediária), exatidão (recuperação).

\section{Seletividade}

A seletividade avalia o grau de interferência de espécies como outro ingrediente ativo, excipientes, impurezas e produtos de degradação, bem como outros compostos de propriedades similares que possam estar, porventura, presentes. ${ }^{18}$ A seletividade garante que o pico de resposta seja exclusivamente do composto de interesse. Para avaliar a seletividade do método, soluções padrão de MDA, MDEA, cafeína, cetamina, cocaína, dextrometorfano, dietilpropiona, efedrina e femproporex $(25 \mu \mathrm{g} / \mathrm{mL})$ foram injetadas no sistema cromatográfico descrito anteriormente. Considerou-se o método como seletivo se não houvesse a presença de picos no mesmo tempo de retenção da MDMA.

\section{Linearidade}

O estudo de linearidade foi conduzido pela injeção em triplicata de 6 concentrações da solução-padrão de MDMA, de 0,2 a $20 \mu \mathrm{g} / \mathrm{mL}$, correspondentes à faixa de $1 \mathrm{a} 100 \%(\mathrm{~m} / \mathrm{m})$ de MDMA nas amostras analisadas, sendo a linearidade estimada através do coeficiente de correlação linear.

\section{Precisão}

Para a determinação da repetibilidade foram analisadas, em triplicata e no mesmo dia, amostras de comprimidos de mesmo grupo. $\mathrm{Na}$ determinação da precisão intermediária foram analisadas, em triplicata e em 3 dias diferentes, amostras de comprimidos pertencentes a um mesmo grupo. Nas duas situações considerou-se como uma "amostra" uma alíquota retirada do pó obtido pela pulverização de um dado lote de comprimidos. Cada amostra aqui mencionada foi preparada do mesmo modo descrito anteriormente.

\section{Exatidão (recuperação)}

A exatidão foi avaliada pelo teste de recuperação analisando-se, em triplicata, amostras de comprimidos de mesmo grupo, adicionadas de quantidades conhecidas de MDMA (em 3 níveis de concentração). 
O percentual de recuperação (exatidão) foi calculado empregando-se a forma proposta pela AOAC. ${ }^{19}$

\section{RESULTADOS E DISCUSSÃO}

O presente trabalho teve por objetivo desenvolver e validar um método analítico para determinar a concentração de MDMA presente nos comprimidos de Ecstasy, além de observar a variação na composição dos comprimidos de um mesmo grupo e grupos diferentes.

A cromatografia líquida de alta eficiência com detecção por fluorescência para determinação de metilenodioximetanfetaminas deve ser considerada técnica de escolha para este tipo de análise, uma vez que estas substâncias são termolábeis e sofrem degradação no bloco de injeção do cromatógrafo a gás. ${ }^{4}$ Para evitar esta degradação, torna-se necessário o uso de reações de derivatização, o que implica no uso de reagentes caros, de significante toxicidade, além de aumentar significativamente o tempo necessário para realização da análise. Deve-se considerar ainda que a CLAE/DF confere ao método alta seletividade e sensibilidade, uma vez que estes analitos são compostos naturalmente fluorescentes. ${ }^{13,14}$

O preparo de amostra, constituído basicamente de dissolução de uma alíquota do comprimido macerado em metanol com posterior diluição em água, foi escolhido por ser um processo simples, de baixo custo, que requer baixo consumo de solvente orgânico.

A identificação do analito foi feita através do tempo de retenção, e a quantificação foi realizada por padronização externa. Como se optou por eluição isocrática, não foi necessária etapa de reequilíbrio do sistema antes da próxima injeção. Assim, o tempo da análise cromatográfica aqui apresentada foi consideravelmente curto (menos de $6 \mathrm{~min}$ ). Metodologias presentes na literatura científica, baseadas em cromatografia gasosa ${ }^{7,20-22}$ e cromatografia líquida ${ }^{23}$ utilizavam pelo menos 7,5 min de corrida cromatográfica.

A partir da injeção de soluções padrão de concentrações conhecidas de MDMA (de 0,2 a $20 \mu \mathrm{g} / \mathrm{mL}$ ), e conseqüente obtenção das respectivas áreas dos picos gerados, foi construída curva analítica que apresentou linearidade satisfatória na faixa de trabalho escolhida, com coeficiente de correlação linear (r) igual a 0,999.

A avaliação da precisão foi calculada através do coeficiente de variação (\%CV), que apresentou valores abaixo de 5\%, tanto nos ensaios de repetibilidade quanto na avaliação da precisão intermediária, estando perfeitamente de acordo para análises forenses. A exatidão do método, avaliada através da recuperação, apresentou valores entre 94 e 104\%. A Tabela 1 apresenta os resultados referentes à precisão e exatidão do método proposto.

Tabela 1. Resultados de precisão e exatidão (recuperação) do método proposto para análise de MDMA em comprimidos de Ecstasy por CLAE-DF

\begin{tabular}{lccc}
\hline \multirow{2}{*}{ MDMA } & \multicolumn{3}{c}{ Níveis de concentração estudados } \\
& $1 \mu \mathrm{g} / \mathrm{mL}^{*}$ & $10 \mu \mathrm{g} / \mathrm{mL}^{*}$ & $20 \mu \mathrm{g} / \mathrm{mL}^{*}$ \\
\hline Repetibilidade (\%CV) & 3,1 & 1,6 & 1,2 \\
Precisão & & & \\
intermediária (\%CV) & 4,2 & 2,1 & 2,7 \\
Exatidão (\%) & 104 & 92 & 101 \\
\hline
\end{tabular}

* cada concentração foi analisada em triplicata.

Vários autores referem que, dentre os principais adulterantes presentes nos comprimidos comercializados no mercado ilícito como sendo Ecstasy estão MDEA, MDA, efedrina, pseudoefedrina, ácido acetilsalicílico, cafeína, dextrometorfano, cetamina, cocaína, dentre outras substâncias. ${ }^{24-27}$ Alguns desses adulterantes podem ser até mais tóxicos que a própria MDMA. Essa variabilidade de composição dos comprimidos e o fato de que usuários de Ecstasy também podem ser consumidores de outras drogas, como maconha, cocaína, alucinógenos e álcool, eleva o risco de intoxicações. Este trabalho não objetivou a identificação de possíveis adulterantes presentes nos comprimidos, mas levou em consideração que algumas substâncias poderiam ser interferentes do método analítico desenvolvido. No teste de seletividade, apenas MDA e MDEA forneceram resposta no detector de fluorescência, contudo em tempos de retenção diferentes do fornecido pela MDMA (Figura 2).

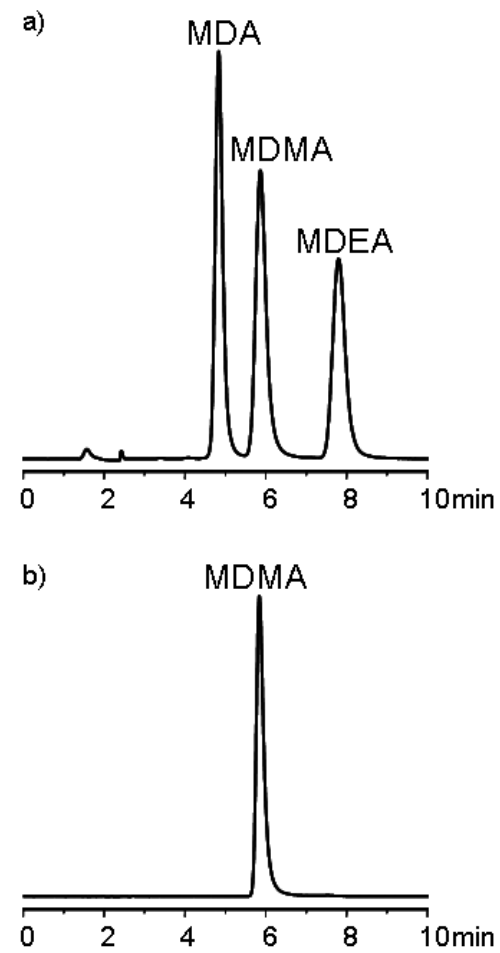

Figura 2. Cromatogramas obtidos pela injeção de mistura de padrões de MDMA e possiveis adulterantes (MDA, MDEA, anfetamina, metanfetamina e cetamina) e de amostra de comprimido de Ecstasy analisado no sistema CLAE/DF. Condições cromatográficas: coluna C-18 (125 x $4 \mathrm{~mm}, 5 \mu \mathrm{m})$; fase móvel: tampão fosfato $25 \mathrm{mM} \mathrm{pH}$ 3,0 e acetonitrila $(95: 5 \mathrm{v} / \mathrm{v}) ; \lambda_{\text {exc. }}=285$ $\mathrm{nm}, \lambda_{\text {emis. }}=324 \mathrm{~nm}$

A dosagem típica para uso recreacional de MDMA presente em um comprimido pode variar de 50 a $150 \mathrm{mg}$, com variações de concentração que podem chegar a $70 \%$ ou mais. ${ }^{28,29}$ Cole et al..$^{25}$ analisaram 136 amostras de comprimidos apreendidos no Reino Unido durante o ano de 2001 e observaram que a quantidade de MDMA presente nestes comprimidos possuiu grande variação (entre 20 e 109 mg de MDMA por comprimido).

Nas amostras analisadas, a concentração de MDMA presente nos comprimidos de Ecstasy apresentou acentuada variabilidade, levando-se em consideração que a massa dos comprimidos não apresenta variação de peso maior que $10 \%$ (entre os comprimidos de mesmo lote). Comprimidos de mesmo grupo apresentaram concentrações de 4,8 a 60,4 (em miligramas de MDMA por comprimido). Comparando-se a concentração de MDMA em comprimidos de lotes diferentes, essa variação também é muito acentuada: a média das concentrações encontrada nos diferentes lotes variou de 8,4 a $59,5 \mathrm{mg}$ por comprimido, conforme os resultados apresentados nas Tabelas 2 a 6. Estas variações nas concentrações devem estar 
Tabela 2. Concentração de MDMA encontrada nos comprimidos do Grupo 1

\begin{tabular}{lcc}
\hline Grupo 1 & Concentração \% (m/m) & Concentração (mg/cp) \\
\hline A & 5,2 & 10,4 \\
B & 9,8 & 19,6 \\
C & 7,9 & 15,8 \\
D & 8,1 & 15,7 \\
E & 6,4 & 12,8 \\
F & 2,5 & 4,8 \\
G & 25,5 & 50,1 \\
H & 22,0 & 41,4 \\
I & 28,3 & 60,4 \\
J & 16,3 & 32,7 \\
K & 26,0 & 51,7 \\
L & 20,0 & 38,5 \\
\hline Média & $\mathbf{1 4 , 8}$ & $\mathbf{2 9 , 5}$ \\
Desvio padrão & $\mathbf{9 , 2 2}$ & $\mathbf{1 8 , 6 7}$ \\
C. V. (\%) & $\mathbf{6 2 , 1}$ & $\mathbf{6 3 , 3}$ \\
\hline
\end{tabular}

Tabela 3. Concentração de MDMA encontrada nos comprimidos do Grupo 2

\begin{tabular}{lcc}
\hline Grupo 2 & Concentração \% (m/m) & Concentração $(\mathrm{mg} / \mathrm{cp})$ \\
\hline $\mathrm{A}$ & 14,6 & 29,0 \\
$\mathrm{~B}$ & 15,0 & 30,3 \\
$\mathrm{C}$ & 16,5 & 31,5 \\
$\mathrm{D}$ & 15,0 & 29,4 \\
$\mathrm{E}$ & 14,8 & 28,2 \\
\hline Média & $\mathbf{1 5 , 2}$ & $\mathbf{2 9 , 7}$ \\
Desvio padrão & $\mathbf{0 , 7 6}$ & $\mathbf{1 , 2 7}$ \\
C. V. (\%) & $\mathbf{5 , 0}$ & $\mathbf{4 , 3}$ \\
\hline
\end{tabular}

Tabela 4. Concentração de MDMA encontrada nos comprimidos do Grupo 3

\begin{tabular}{lcc}
\hline Grupo 3 & Concentração \% (m/m) & Concentração $(\mathrm{mg} / \mathrm{cp})$ \\
\hline A & 3,1 & 7,7 \\
B & 1,0 & 2,6 \\
C & 1,0 & 2,6 \\
D & 2,3 & 5,9 \\
E & 12,2 & 30,4 \\
F & 12,1 & 30,4 \\
G & 8,0 & 20,2 \\
H & 4,9 & 12,6 \\
\hline Média & $\mathbf{5 , 6}$ & $\mathbf{1 4 , 1}$ \\
Desvio padrão & $\mathbf{4 , 6 3}$ & $\mathbf{1 1 , 6 0}$ \\
C. V. $(\boldsymbol{\%})$ & $\mathbf{8 2 , 7}$ & $\mathbf{8 2 , 3}$ \\
\hline
\end{tabular}

Tabela 5. Concentração de MDMA encontrada nos comprimidos do Grupo 4

\begin{tabular}{lcc}
\hline Grupo 4 & Concentração \% (m/m) & Concentração (mg/cp) \\
\hline A & 3,2 & 8,1 \\
B & 3,2 & 8,2 \\
C & 3,6 & 9,2 \\
D & 3,5 & 8,5 \\
E & 3,4 & 8,5 \\
F & 3,5 & 8,7 \\
G & 3,5 & 8,4 \\
H & 3,5 & 8,9 \\
I & 3,2 & 7,9 \\
J & 3,2 & 7,9 \\
\hline Média & $\mathbf{3 , 4}$ & $\mathbf{8 , 4}$ \\
Desvio padrão & $\mathbf{0 , 1 6}$ & $\mathbf{0 , 4 2}$ \\
C. V. (\%) & $\mathbf{4 , 8}$ & $\mathbf{5 , 0}$ \\
\hline
\end{tabular}

Tabela 6. Concentração de MDMA encontrada nos comprimidos do Grupo 5

\begin{tabular}{lcc}
\hline Grupo 5 & Concentração \% $(\mathrm{m} / \mathrm{m})$ & Concentração $(\mathrm{mg} / \mathrm{cp})$ \\
\hline A & 20,3 & 62,5 \\
B & 21,4 & 66,1 \\
C & 20,0 & 58,7 \\
D & 22,2 & 65,2 \\
E & 21,1 & 64,4 \\
F & 21,5 & 65,4 \\
G & 12,4 & 35,4 \\
H & 13,0 & 39,7 \\
I & 20,7 & 63,0 \\
J & 21,3 & 67,1 \\
K & 20,9 & 62,2 \\
L & 20,3 & 63,7 \\
\hline Média & $\mathbf{1 9 , 6}$ & $\mathbf{5 9 , 5}$ \\
Desvio padrão & $\mathbf{3 , 2 8}$ & $\mathbf{1 0 , 4 9}$ \\
C. V. (\%) & $\mathbf{1 6 , 7}$ & $\mathbf{1 7 , 6}$ \\
\hline
\end{tabular}

relacionadas ao fato de não existir controle na produção dos comprimidos, sendo que processos farmacotécnicos simples, como a homogeneização do ingrediente ativo com os excipientes, foram pouco eficientes.

Os comprimidos analisados mostraram concentrações de MDMA inferiores a outros comprimidos analisados em anos anteriores por outros autores, como Lapachinske et al., em 2004, ${ }^{7}$ que observaram variação entre 30,9 e 92,7 mg por comprimido. Cumpre enfatizar que esse fato, além de fatores como farmacodependência e tolerância, pode estar relacionado ao aumento do consumo de Ecstasy pelos usuários, que chegam a ingerir vários comprimidos numa festa.

Como esperado, a variação da concentração de MDMA em comprimidos de lotes diferentes é ainda mais pronunciada, o que pode estar relacionado aos modos de preparo dos comprimidos por diferentes laboratórios clandestinos. 
Deve-se considerar que esta grande variação na quantidade de MDMA presente nos comprimidos pode, em conjunto com fatores como farmacodependência e tolerância, estar relacionada com o aumento do consumo de comprimidos de Ecstasy por usuários, que chegam a ingerir vários comprimidos numa festa, inconscientemente suprimindo a baixa dosagem presente nos comprimidos. Se houver mudança no fornecedor, para comprimidos mais concentrados em MDMA e se o usuário mantiver o mesmo padrão de uso, uma superdosagem pode ocorrer, aumentando o risco de intoxicações. ${ }^{7}$

\section{CONCLUSÕES}

O método de análise de 3,4-metilenodioximetanfetamina em comprimidos de Ecstasy por cromatografia líquida de alta eficiência com detecção por fluorescência mostrou-se rápido, linear, específico, preciso e exato. Por utilizar reagentes e procedimentos simples, o método apresenta-se como uma alternativa para a quantificação desta substância ativa proscrita na droga de abuso apreendida, podendo ser implantado com facilidade em laboratórios forenses de todo Brasil.

Pela análise de amostras encaminhadas para exame pericial, foi constatado que existe significativa variação na quantidade de MDMA nos comprimidos de Ecstasy. Este fato, que certamente aumenta o risco de intoxicações agudas, é de grande relevância para que se entenda a epidemiologia das intoxicações por Ecstasy.

\section{REFERÊNCIAS}

1. Nichols, D. E.; J. Psychoact. Drugs 1986, 18, 305.

2. http://www.unodc.org/unodc/en/data-and-analysis/WDR-2008.html, acessada em Setembro 2008.

3. Landry, M.; J. Psychoactive Drugs 2002, 34, 163.

4. Gimeno, P.; Besacier, F.; Chaudron-Thozet, H.; Forensic Sci. Int. 2003, $132,182$.

5. Silva, O. A.; Yonamine, M.; Reinhardt, V. E.; Rev. Farm. Bioquím. Univ. São Paulo 1998, 34, 33.

6. Baptista, M. C.; Noto, A. R.; Nappo, S.; Carlini, E. A.; J. Bras. Psiquiatria 2002, 51, 81.

7. Lapachinske, S. F.; Yonamine, M.; Moreau, R. L. D. M.; Rev. Bras. Ciências Farm. 2004, 40, 75.

8. http.//www.dpf.gov.br, acessada em Maio 2008.
9. Green, R.; Mechan, A. O.; Elliot, J. M.; O'shea, E.; Colado, I.; Pharmacol. Rev. 2003, 55, 463.

10. Ferigolo, M.; Medeiros, F. B.; Barros, H. M. T.; Rev. Saúde Pública 1998, 32,487

11. Brunnenberg, M.; Kovar, K. A.; J. Chromatogr., B: Anal. Technol. Biomed. Life Sci. 2001, 751, 9.

12. Buechler, J.; Schwab, M.; Mikus, G.; Fischer, B.; Hermle, L.; Marx, C.; Gron, G.; Spitzer, M.; Kovar, K. A.; J. Chromatogr., B: Anal. Technol. Biomed. Life Sci. 2003, 793, 207.

13. Clauwaert, K. M.; Bocxlaer, J. F. V.; Leenheer, A. P.; Forensic Sci. Int. 2001, 124, 36

14. Clauwaert, K. M.; Van Bocxlaer, J. F.; De Letter, E. A.; Van Calenbergh, S.; Lambert, W. E.; De Leenheer, A. P.; Clin. Chem. 2000, 46, 1968.

15. Meyer, A.; Mayerhofer, A.; Kovar, K. A.; Schmidt, W. J.; Neurosci. Lett. 2002, 330, 193.

16. Peters, F. T.; Maurer, H. H.; Accred. Qual. Assur. 2002, 7, 441.

17. http://www.soft-tox.org/docs/Guidelines $\% 202006 \% 20$ Final.pdf, acessada em Maio 2008.

18. Ribani, M.; Bottoli, C. B. G.; Collins, C. H.; Jardim, I. C. S. F.; Melo, L. F. C.; Quim. Nova 2004, 27, 771.

19. AOAC, Association of Analytical Chemists; Official Methods of Analysis, $15^{\text {th }}$ ed., AOAC: Arlington, 1990.

20. Han, E.; Yang, W.; Lee, J.; Park, Y.; Kim, E.; Lim, M.; Chung, H.; Forensic Sci. Int. 2005, 152, 73.

21. Lasmar, M. C.; Leite, E. M. A.; Rev. Bras. Ciências Farm. 2007, 43, 223.

22. Villamor, J. L.; Bermejo, A. M.; Fernández, P.; Tabernero, M. J.; J. Anal. Toxicol. 2005, 29, 135.

23. Concheiro, M.; Castro, A. D.; Quintela, O.; Lopez-Rivadulla, M.; Cruz, A.; Forensic Sci. Int. 2005, 150, 221.

24. Baggott, M.; Heifets, B.; Jones, R. T.; Mendelson, J.; Sferios, E.; Zehnder, J.; J. Am. Med. Assoc. 2000, 284, 2190.

25. Cole, J. C.; Bailey, M.; Sumnall, H. R.; Wagstaff, G. F.; King, L. A.; Addiction 2002, 97, 1531

26. Silva, O. A.; Yonamine, M.; Reinhardt, V. E. D.; Rev. Farm. Bioquim. Univ. São Paulo 1998, 34, 33.

27. Sherlock, K.; Wolff, K.; Hay, A. W.; Conner, M.; J. Accid. Emerg. Med. 1999, 16, 194.

28. Laranjeira, R.; Dunn, J.; Rassi, R.; Fernandes, M.; Rev. ABP-APAL 1996, $18,77$.

29. Kalant, H.; Can. Med. Assoc. J. 2001, 165, 917. 